This item was submitted to Loughborough's Research Repository by the author.

Items in Figshare are protected by copyright, with all rights reserved, unless otherwise indicated.

\title{
Physics of ultra-thin surface films on molecularly smooth surfaces
}

PLEASE CITE THE PUBLISHED VERSION

PUBLISHER

Professional Engineering Publishing / @ IMECHE

VERSION

VoR (Version of Record)

LICENCE

CC BY-NC-ND 4.0

REPOSITORY RECORD

Teodorescu, M., Sashi Balakrishnan, and Homer Rahnejat. 2019. "Physics of Ultra-thin Surface Films on Molecularly Smooth Surfaces”. figshare. https://hdl.handle.net/2134/4730. 
This item was submitted to Loughborough's Institutional Repository (https://dspace.lboro.ac.uk/) by the author and is made available under the following Creative Commons Licence conditions.

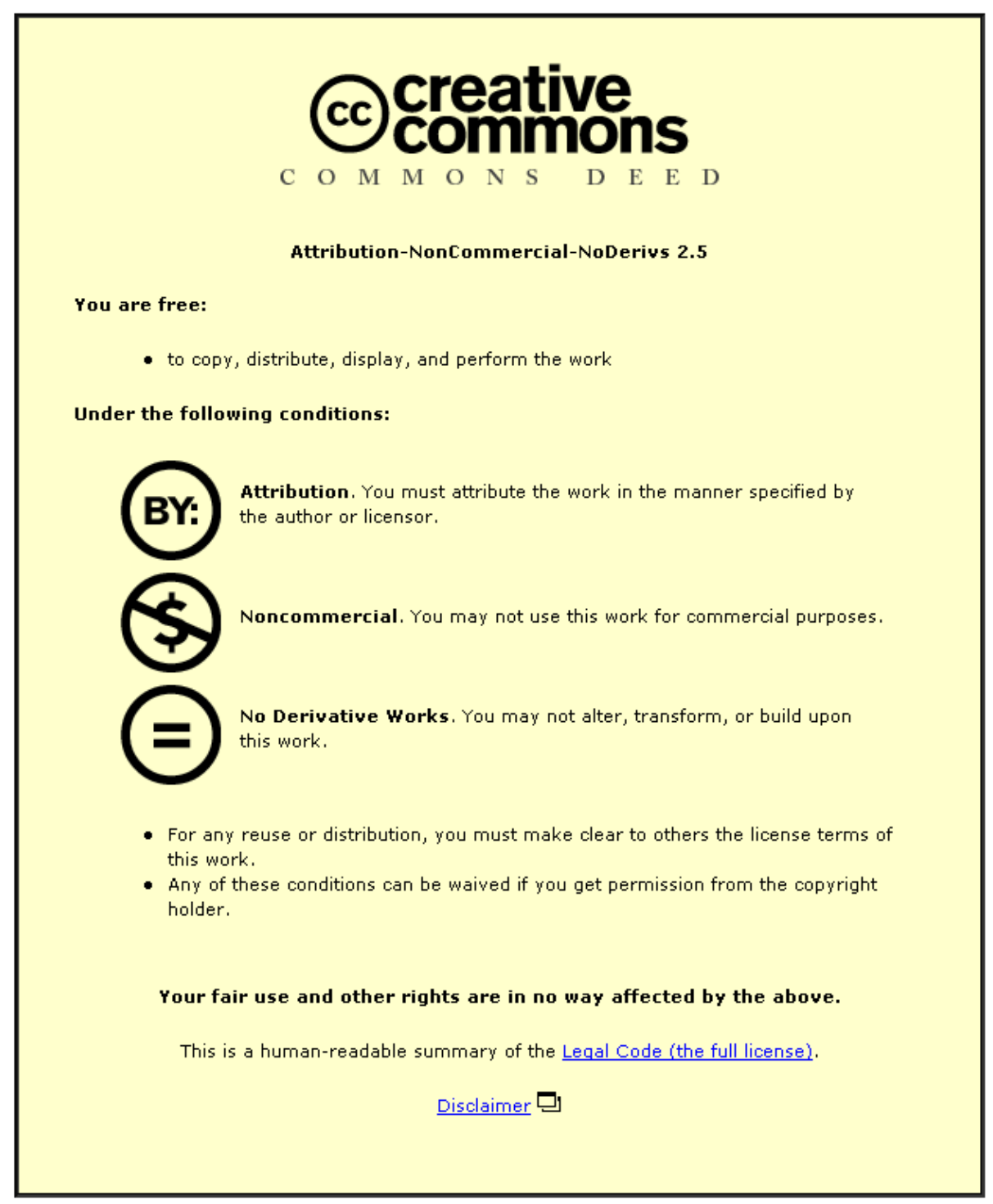

For the full text of this licence, please go to: http://creativecommons.org/licenses/by-nc-nd/2.5/ 


\title{
Physics of ultra-thin surface films on molecularly smooth surfaces
}

\author{
M Teodorescu $^{1}$, S Balakrishnan ${ }^{2}$, and H Rahnejat ${ }^{2 *}$ \\ ${ }^{1}$ School of Engineering and Mathematical Sciences, City University, London, UK \\ ${ }^{2}$ Wolfson School of Mechanical and Manufacturing Engineering, Loughborough University, Loughborough, UK
}

The manuscript was received on 12 June 2006 and was accepted after revision for publication on 25 October 2006.

DOI: $10.1243 / 17403499 J N N 59$

\begin{abstract}
This paper investigates the physics of ultra-thin surface films in nano-scale counterformal conjunctions. A novel approach is proposed in which the transient behaviour of the fluid film is integrated with the contact mechanics of the approaching bodies. The method predicts film thickness and pressure distribution, as well as local elastic deflection and resulting sub-surface stress tensor. It is found that inertial dynamics of bodies, as determined by local squeeze film effect, prevents diminution of film below certain thickness and reduces the solvation effect. This approach has direct applications for micro-scale mechanisms, where prediction of thin surface adhered film thickness is required for tribological purposes, as well as structural in-service integrity of contacts of vanishing dimensions.
\end{abstract}

Keywords: hydrodynamics, solvation force, van der Waals force, ultra-thin fluid films, subsurface stress tensor

\section{INTRODUCTION}

Progressively many engineering surfaces are covered with ultra-thin films of molecular dimensions, creating vanishingly small gaps. Applications range from data storage devices to microelectro-mechanical systems (MEMS). The behaviour of these thin films is not in accord with the well-understood theories of physics, such as the Newtonian slow viscous action (hydrodynamics) [1], the Lifshitz theory of van der Waals forces [2, 3] or electrostatic double layer interactions for charged surfaces (known as the DLVO theory) $[\mathbf{4}, \mathbf{5}]$. The Lifshitz theory predicts a monotonically attractive force between any two bodies separated by a third medium, the DLVO theory predicts a repulsive force between such bodies, and hydrodynamics predicts the separation of surfaces, when subjected to relative motion, all based on the bulk properties of the intervening film. The problem with increasing miniaturization of devices is that the discrete nature of molecular interactions at ultra-thin separations is unaccounted for in these

*Corresponding author: Wolfson School of Mechanical and Manufacturing Engineering, Loughborough University, Loughborough, LE11 3TU, UK. email: h.rahnejat@lboro.ac.uk theoretical predictions. In other words, the kinetics described by these force laws refer to the existence of a continuum, which is not upheld by the observed discrete behaviour of films of molecular dimension. The initial, surprising findings of the deviant behaviour of ultra-thin films was put at the doorstep of a breakdown in the DLVO theory for the so-called cases of 'structureless molecular behaviour'. However, it has become clear in recent years that the behaviour of discrete molecules can be explained in terms of ultra-thin film surrounding effects of a structural nature, now invariably referred to as solvation [6-8].

It has become clear that molecular density of the film deviates from its bulk value in an oscillatory fashion, which accounts for the structural (solvation) force, the magnitude of which exceeds that of the conventional van der Waals force predicted by the Lifshitz theory for films of the order of several molecular diameters of the intervening fluid [6]. As the thickness of the film increases further, its density eventually reaches its bulk value, and the kinetic behaviour merges into the attractive van der Waals force.

The solvation effect has a profound implication for many narrow load-bearing conjunctions, as well as 
the docking of molecules onto solid barriers, such as in the attachment of proteins to surfaces in biological systems. Often, in bearings, an ideal surface film is envisaged to protect the mating surfaces against wear damage. At the interface with such solid surfaces, the thin films are subject to attractive interactions with the solids, which also impose a constraining effect, thereby regimenting the film molecules into quasi-discrete layers, with an oscillatory density profile. This usually leads to a denser packing of film molecules near the solid surfaces [9]. The effect is particularly pronounced near molecularly smooth surfaces. Thus, on the face of it, the solvation effect should indeed provide the level of protection that nano-level conjunctions urgently need. With devices such as MEMS, one common problem is the ingression of moisture into contacts, which can have the adverse effect of forming nanolevel menisci, the repercussion being the adhesion of surfaces at very close separations (usually of the order of a couple of water molecular diameters) $[10,11]$. A preventative action is to attempt to produce thin molecular films of a hydrophobic nature, which, by virtue of larger density distribution near the solid walls and an ultra-thin nature (often of a mono-layer structure), promote the solvation effect and inhibit surface-to-surface attractions $[12,13]$. However, surface smoothness cannot yet be assured in any significant manner in, for example, MEMS devices and the solvation effect can remain rather partial (interrupted). Furthermore, mutual convergence of load-bearing pairs often re-order the molecular layers, leading to the drainage of molecules in a discrete fashion [14]. Observation of this phenomenon has led to the empirical formulation of the solvation effect, and, more importantly, the reduction of layer levels (usually to a limit of two to three molecular levels) can lead to the eventual dominance of 'microscopic level' meniscus force, thus the onset of at least localized stiction $[\mathbf{1 5}, \mathbf{1 6}]$.

On the other hand, an excessive number of quasidiscrete molecular layers can lead to the diminution of the solvation effect by the formation of a hydrodynamic film, particularly with any entraining of the film by relative motion of the load-bearing surfaces. This improves the opportunity for the formation of 'macroscopic level' menisci, particularly at instances of very low relative surface velocities or motion reversals (diminished hydrodynamic action). Under these circumstances, a balance between the meniscus force and electrostatic repulsion (often used as the driving force for micromechanisms) can lead to forced contact breaking action and ensuing structural damage [17].

It, therefore, appears that a compromise solution may lie in the maintenance of ultra-thin discretized films of certain molecular dimensions above the limit of adhesion that would be governed by ingression of moisture without reliance on hydrodynamic entrainment. Such an opportunity can only exist by squeeze-film effect within a range that would limit surface molecular density to a prescribed minimum level. This paper is an attempt to explore this proposition by simultaneous solution of hydrodynamic, solvation, and van der Waals kinetic action for the general case of a line of contact of a pair of constraining walls under mutual approach and separating motions.

\section{THEORETICAL FORMULATION}

Ultra-thin films in conjunctions between constraining surfaces in relative combined entraining and squeeze-film motions with low applied loads and very small contact areas are subject to complex kinetic interactions, which can be described by the various force laws listed above. The interactions between these also play important roles $[\mathbf{7}, \mathbf{8}, \mathbf{1 8}$. There is, in fact, a plethora of kinetic actions, and the purpose of the analysis is best served by reducing the multiplicity of such interactions for certain physically coherent conditions. In this paper, the case of the line contact of two constraining elastic solids is considered. The surfaces are regarded as not possessing any charge with an intervening insulating nonpolar, non-liquid-crystalline thin surface film, such as octamethylcyclotetrasiloxane (OMCTS) or perfluoropolyether. The tribological conditions in such conjunctions are governed, in the main, by mechanisms of hydrodynamic lubrication: entraining and squeeze-film actions, when the bulk fluid properties give rise to a continuum, which is maintained by certain kinematic conditions (chiefly, sufficient relative surface velocities) and separations greater than several molecular diameters of the intervening fluid. At the onset of cessation of relative motion, depletion of surface film occurs and, depending on surface topography, and solid-solid, solid-vapour, and liquid-vapour interfaces, meniscus action can become dominant, leading to stiction in the conjunction, by what one may define as 'macroscopic level surface tension effect'. This problem can be overcome by application of a repulsive action, at least equal to the meniscus force [14]. This, in effect, is a form of increasing the load-carrying capacity of the conjunction, rather similar to that achieved by a greater separation of a contact at a given load by the introduction of a squeeze-film effect. Equilibrating the meniscus action at the so-called 'macroscopic level' in this manner enables the emergence of molecular interactions between the constraining solid boundaries at progressively lower separations, if they are molecularly smooth [19] and inhibit 
meniscus action in such vanishing gaps by what one may term as 'nano-level (microscopic)' menisci. As surface separation is reduced further, the solvation effect, as described above, can become gradually dominant at low surface velocities, although some contributions must remain from hydrodynamics. van der Waals force may also contribute between molecules of the film and the adjacent solids by surface energy effect. Thus, for the special case described here, prediction of kinetic interactions may reasonably be confined to those of low speed, low load hydrodynamics, solvation, and Van der Waals force.

\subsection{Full solution for contact pressure and local deflection}

Therefore, the total pressure in the narrow conjunction is generated by contributions from a number of physical phenomena, described above. Disregarding the effect of meniscus action and considering lightly loaded conjunctions of molecular-level separation

$$
P=P_{h}+P_{s}+P_{w}
$$

the superposition of all the pressure-generating mechanisms (hydrodynamic, solvation, and van der Waals) is justified by the fact that the load carried by the film is supported by all these mechanisms simultaneously. This is the basis of Bernoulli's principle of superposition, which is used for such conjunctions by Israelachvili, Matsuoka and Kato, and Abd-AlSamieh and Rahnejat [6, 7, 18].

With relative motion of constraining surfaces, the hydrodynamic viscous action is the source of delivery of the intervening fluid by combined entraining and squeeze-film motions. The hydrodynamic pressure is obtained by solution of Reynolds equation

$$
\frac{\partial}{\partial x}\left(\frac{\rho_{\infty} h^{3}}{\eta} \frac{\partial P_{h}}{\partial x}\right)=12\left\{u_{a v} \frac{\partial\left(\rho_{\infty} h\right)}{\partial x}+\frac{\partial\left(\rho_{\infty} h\right)}{\partial t}\right\}
$$

This form of the equation represents an infinite line contact, for example, for a roller near a semiinfinite elastic half-space. Since for hydrodynamic contribution a continuum is assumed, boundary conditions are required for solution of equation (2), these being Reynolds boundary conditions as

$$
\left.P_{h}\right|_{X_{\text {inlet }}}=\left.\frac{\partial P_{h}}{\partial x}\right|_{\text {inlet }}=0 \quad \text { and }\left.\quad P_{h}\right|_{x_{\text {outlet }}}=\left.\frac{\partial P_{h}}{\partial x}\right|_{x_{\text {outlet }}}=0
$$

To compute the hydrodynamic pressure $P_{k}$ from equation (2), one needs to determine the bulk rheological state of the fluid: viscosity and density variation with pressure, assuming isothermal conditions here. The lubricant viscosity as a function of pressure is given by Roelands [20] as

$$
\eta=\eta_{0} \exp \left[\ln \eta_{0}+9.67\right]\left[\left(1+5.1 \times 10^{-9} P_{h}\right)^{Z}-1\right]
$$

where $Z=\alpha /\left(5.1 \times 10^{-9}\left[\ln \eta_{0}+9.67\right]\right)$.
The bulk density is given by Dowson and Higginson [21] as

$$
\rho_{\infty}=\rho_{0 \infty}\left(1+\varepsilon P_{h} /\left(1+\xi P_{h}\right)\right)
$$

In very small gaps, the variation of this density near the surface of the solids gives rise to the solvation effect. As a first approximation, the solvation pressure may be considered as an exponentially decaying cosine function $[\mathbf{8}, 14,18]$, which in the limit of diminishing surface separation (i.e. $h \rightarrow 0$, last layer of solvent molecules having been drained out of the contact) approaches a finite value, given by $C=-k T \rho_{\infty}$ [6]. The solvation pressure can thus be given as

$$
P_{s}=-C e^{-h / a} \cos (2 \pi h / a)
$$

where the molecular diameter and the constant $C$ for the type of fluid used must be determined. The results shown here are for typical non-polar spherical molecules, such as octamethylcyclotetrasiloxane and perfluoropolyether, where, typically, $a=1 \mathrm{~nm}$ and $C=172 \mathrm{MPa}$. Note the oscillatory sinusoidal nature of the solvation pressure. The exponential decaying cosine nature of solvation pressure merits investigation as a transitory pressure wave through the contact, which yields some interesting physical understanding. Equation (6) can be written in the equivalent form

$$
P_{s}(h)=C_{f} e^{-\frac{1}{a} h} \cos \left(\frac{2 \pi}{a} h-\pi\right)
$$

where $\pi$ indicates the phase angle. Like all oscillatory motions, this represents the solution to the equation of motion of the form

$$
\frac{\partial^{2} P_{s}(h)}{\partial h^{2}}+2 \zeta \omega_{n} \frac{\partial P_{s}(h)}{\partial h}+\omega_{n}^{2} P_{s}(h)=0
$$

where, from elementary theory, $\omega_{d}=\omega_{n} \sqrt{1-\zeta^{2}}=$ $2 \pi / a$ and $\zeta \omega_{n}=1 / a$, thus

$$
\omega_{n}=\frac{\sqrt{4 \pi^{2}+1}}{a} \text { and } \zeta=\frac{1}{\sqrt{4 \pi^{2}+1}}
$$

Now, the period of pressure wavefront oscillations is $2 \pi / \omega_{d}=a$, which is in line with the observations $[\mathbf{6}, 22]$. An interesting feature of the analysis is that $\zeta$ remains independent of the fluid molecule, indicating an exponential decay rate in the discretization process. This translates to exponentially decaying discrete energy levels (integer powers of $e$ ) with increasing molecular layers, which eventually merges into the continuum determined by the DLVO or van der Waals interfacial energy. This means that the layering process only occurs at certain energy levels (or applied contact forces), as shown by Abd-AlSamieh [11], a form of quantized behaviour. It is also noted 
that the effect of solvation is not strictly additive with that of van der Waals interactions, as suggested by equation (1). However, when dealing with analysis of decreasing molecular levels of separation, the boundary where either form of interaction becomes significant depends on the properties of the intervening fluid, such as density, refractive index, and relative permittivity. Where one phenomenon is dominant, the other is almost insignificant.

The van der Waals interaction energy between two surfaces separated by a very thin fluid film can be obtained, taking into account the interactions between the atoms of the two surfaces, and these with the molecules of the intervening fluid film [2]. Since the contact area is usually very small in nano-conjunctions, such as those described here, one can assume the interaction energy to act between two flat parallel planes [18]. Thus

$$
P_{w}=-\frac{A_{131}}{6 \pi h^{3}}
$$

where in general $A_{131} \cong 10^{-20}-10^{-19} \mathrm{~J}$.

An order of magnitude analysis of the above pressure-generating sources would indicate that at higher values of entraining velocity (i.e. larger separations) the hydrodynamic effect would dominate the physics of the conjunction. This is also true of decreasing applied loads. Otherwise, the separation reduces and the constraining effect becomes significant. It is surmised that this would lead to the dominance of structural solvation. Another important observation is that the negative generated pressures, either by attractive van der Waals interactions or local density variation by solvation, tend to bring the surfaces together. These reduce the load-carrying capacity of the conjunction. The load carried by the conjunction is

$$
W=\int P d x
$$

where pressure, $P$, is given by equation (1). The few conventional solutions that are reported for such gaps of molecular dimension presuppose that the viscous action of the fluid would result in a hydrodynamic conjunction, which determines an initial gap size, which in turn may be disrupted by the discrete molecular behaviour, if of certain levels of separation $[\mathbf{7}, \mathbf{8}, \mathbf{9}, \mathbf{1 8}]$. This approach is based on the philosophy that the hydrodynamic action strives to form a continuum, which may not be sustained for given loads and kinematic conditions. In such an approach, numerical convergence for pressure generation is based on the conventional hydrodynamic solutions, which is then moderated by load convergence, taking into account other kinetic interactions. The approach reduces the computational burden, and comparisons with measured data show good degrees of conformance.
However, the underlying philosophy is not entirely coherent, in that a continuum cannot be assumed as pre-existing, only to be interrupted by prevailing sources of discretization at a subsequent stage. In the current analysis, convergence for total conjunctional pressure is undertaken as shown in the flow chart of Fig. 1(a) for a set of pre-described competing kinetics within the same environment. This amounts to a closed field concept, but has the drawback of computational complexity. The pressures $P_{h}, P_{s}$, and $P_{w}$ are deemed as converged when the convergence criterion

$$
\sum_{x_{\text {inlet }}}^{x_{\text {outet }}}\left\{\left|P_{n}(x)\right|-\left|P_{n-1}(x)\right|\right\} / \sum_{x_{\text {inlet }}}^{x_{\text {outet }}} P_{n}(x) \leq 10^{-5}
$$

(where $n$ refers to the iteration number) is satisfied.

Another improvement upon the reported solutions is the inclusion of microscopic localized effects. This is achieved by an implicit consideration of the attractive nature of both the solvation effect and van der Waals interactions that would tend to further promote the mutual approach of the constraining walls, rather than opposing it in a localized manner. In other words, they can reduce the loadcarrying capacity of the conjunction. When the constraining walls are of a solid nature, the negative suction effect may be of no consequence and its omission is thus justified as already reported. However, the same cannot be assumed for those of a delicate nature, such as in the MEMS devices.

For an infinite line contact, representing a roller near a semi-infinite elastic half-space, the hydrodynamic pressure distribution is parabolic in the transverse direction, which is the direction of entraining motion of the fluid into the contact area. The axial pressure distribution is considered to be uniform. In conventional contacts, as the pressure is increased, the hydrodynamic regime of lubrication gives way to elastohydrodynamics, where the surface deformation of the contiguous bodies in contact contributes to the gap size filled by the fluid. In contrast, in lightly loaded contacts of molecularly smooth surfaces of relatively high elastic modulus, such as mica or silica, any contact deformation is found to be due to the structural solvation force with separations of the order of a few molecular diameters of the intervening fluid [7]. In any case, the eventual gap size, referred to as the elastic film shape, is as a result of the rigid (undeformed) separation, determined by the geometry of the surfaces (profile), together with any local deformation (deflection). The elastic film profile is expressed as

$$
h=h_{\text {ref }}+s+\delta
$$

The local deflection for a line of contact is computed at each location within the contact as 
(a)

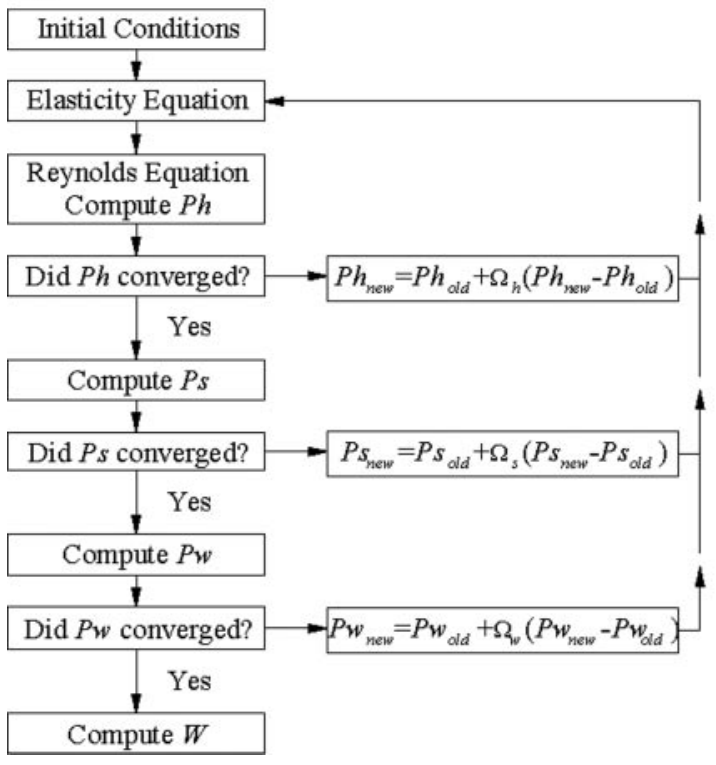

(b)

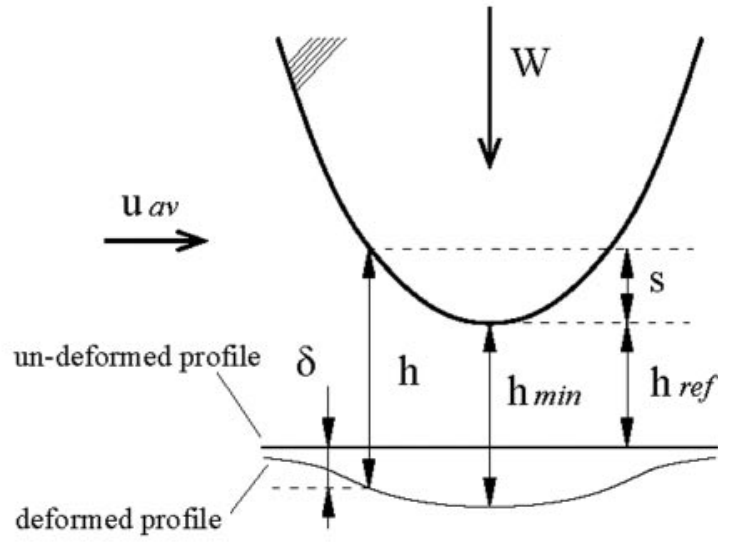

Fig. 1 (a) Computational flow chart and (b) geometry of elastic film shape

the weighted sum of all pressure elements in the same manner as that described by Hamrock [23]

$$
\delta(x, t) \approx \sum_{x^{*}=x_{\text {inlet }}}^{x_{\text {outlet }}} P\left(x^{*}, t\right) D\left(x, x^{*}\right)
$$

where $D\left(x, x^{*}\right)$ is the influence coefficient given [23].

\subsection{Sub-surface stress field calculation}

Two mechanisms of failure would play important roles in the case of ultra-thin film conjunction of molecularly smooth surfaces of the kind described by the current theoretical formulation. Firstly, the diminution of the surface film results in an effective line load determined by meniscus action, with a limit of two molecular diameters of the intervening fluid in most cases, shown to be the case for OMCTS [11]. This, therefore, is the limit of adhesion, determined by the meniscus force

$$
F_{m}=\frac{4 \pi \gamma_{L} \cos \theta}{1+\frac{h}{d}}
$$

where, clearly, $h / d<<1$.

In fact, Christenson [24], using the same equation, showed that small amount of moisture between constraining mica surfaces can dramatically enhance the chance of adhesion. The current paper does not include the effect of meniscus action and stiction, and the subsequent contact breaking (wear and surface tears).

In concentrated contacts of non-conforming surfaces, such as a roller against a flat plane (described in this paper), the pressure distribution induces stresses beneath the surfaces of contacting bodies. These are often a determining factor in the inelastic deformation there, leading to pitting of surfaces. This phenomenon is referred to as fatigue spalling, which together with wear represents the significant potential failure mechanisms. Here, the cyclic stressing of the contact by oscillatory transient behaviour is shown, which is mainly governed by surface energy effect rather than by hydrodynamic pressure loading, which occurs in medium to heavy loaded bearing conjunctions. In order to observe this deviant behaviour from continuum mechanics in a computationally efficient manner, an analytical pressure decomposition technique is developed.

By virtue of Bernoulli's principle of superposition, the total surface and sub-surface stresses and strains at any location can be expressed as $[\mathbf{2 5}, \mathbf{2 6}]$

$$
\sigma_{i j}={ }_{0} \sigma_{i j}+\sum_{k=1}^{N \rightarrow \infty}{ }_{k} \sigma_{i j}, \quad i, j \in\{x, y\}
$$

The contact pressure, giving rise to the abovementioned stress tensor, can then be decomposed into a Fourier series as

$$
P=\frac{1}{2} P_{0}+\sum_{k=1}^{\infty} P_{k} \cos \left(\frac{2 \pi x}{\lambda_{k}}-\varphi_{k}\right)
$$

The sub-surface contact stress field generated by the constant term component of the stress tensor due to the average pressure (i.e. ${ }_{0} \sigma_{i j} ; i, j \in\{x, y\}$ ) can be computed using the classical theory $[\mathbf{2 7}, \mathbf{2 8}]$.

For a generic $k^{\text {th }}$ pressure harmonic order of the same tensor, the sub-surface stress field can be expressed as $[\mathbf{2 5}, \mathbf{2 6}]$ 


$$
\left\{\begin{array}{l}
{ }_{k} \sigma_{x x} \operatorname{sech} \alpha_{k} y=P_{k} \cos \left(\alpha_{k} x-\varphi_{k}\right)\left(\alpha_{k} y-1\right)\left(\tanh \alpha_{k} y-1\right) \\
{ }_{k} \sigma_{y y} \operatorname{sech} \alpha_{k} y=-P_{k} \cos \left(\alpha_{k} x-\varphi_{k}\right)\left(\alpha_{k} y+1\right)\left(\tanh \alpha_{k} y-1\right) \\
{ }_{k} \sigma_{x y} \operatorname{sech} \alpha_{k} y=P_{k} \alpha_{k} \sin \left(\alpha_{k} x-\varphi_{k}\right) y\left(1-\tanh \alpha_{k} y\right)
\end{array}\right.
$$

\section{RESULTS AND DISCUSSIONS}

When two bodies are made to mutually approach each other (as is proposed here by successive reduction in the minimum rigid gap, $h_{r e f}$ ), a corresponding pressure is generated in the intervening fluid by the mechanism known as the squeeze-film effect. If the analysis is carried out ignoring this residual effect, the analysis of the approaching surfaces is taken into account as a series of quasi-static solutions. Here, this approach is referred to as 'steady state'. On the other hand, when the continuity of action from one separation to another is included in the analysis, the time history of film behaviour is retained. This is referred to as 'transient'.

\subsection{Steady state}

To gain an initial appreciation of molecular discretization and fluid film layering effect, a series of steady state solutions at different solid surface separations is undertaken. Since each separation level is considered independent of a preceding one, the historical squeeze-film effect is disregarded $(\partial(\rho h) / \partial t=0)$, thus the analysis cannot be regarded as quasi-static. The rigid steady state film (i.e. the minimum separation is determined by the profile of the roller near the semi-infinite flat half-space) is denoted by $h_{0 \mid x=0}$. This minimum rigid separation is hereinafter referred to as $h_{r e f}$. The kinetic interactions in such a gap cause an elastic film, comprising the undeformed profile and the initial separation of the bodies, as well as any surface deflection, as indicated by equation (11). The minimum separation is now denoted by $h_{\text {min }}$.

Figure 2(a) shows a plot of computed values of $h_{\text {min }}$ for a series of predetermined separations $h_{r e f}$. At larger separations, the dominance of hydrodynamic action leads to a linear variation of slope of unity, as shown by the computed values conforming to the indicated linear slope (the dotted line: the undeformed case). This is anticipated and indicates continuum iso-viscous rigid contact condition. With $h_{\text {ref }}$ below several molecular diameters of the fluid, the constraining effect caused by structural confinement begins to initiate discrete molecular behaviour, and deviation from the linearity is observed. Below this level, the rising contribution due to solvation manifests itself in the layering effect in the minimum film thickness. This represents the discrete nature of fluid drainage from such confine-
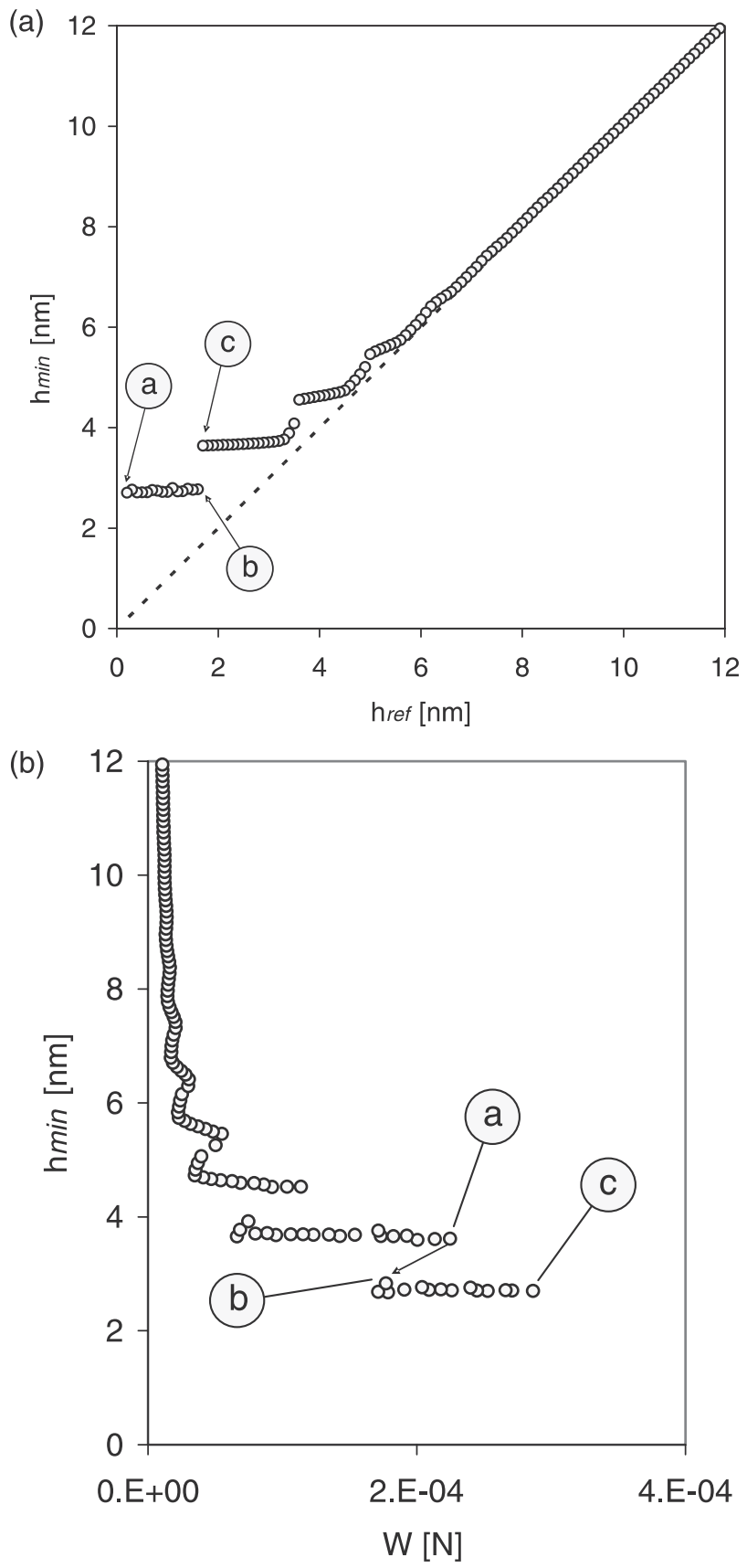

Fig. 2 Computed molecular discretization and fluid film layering effect (for three values of the separation $-\mathrm{a}, \mathrm{b}$, and $\mathrm{c}-$ the oil film thickness is shown in Fig. 3 and the corresponding pressures in Fig. 4.) (a) minimum film thickness as a function of separation, (b) contact load

ment, which was observed by Chan and Horn [14], and which is not in accord with the expected continuum hydrodynamic viscous action described by the Newtonian model. Figure 2(b) shows the same overall picture in terms of the integrated total pressure or the load-carrying capacity of the conjunction. An increasing load is reciprocally proportional to a decreasing film in a dominantly hydrodynamic 


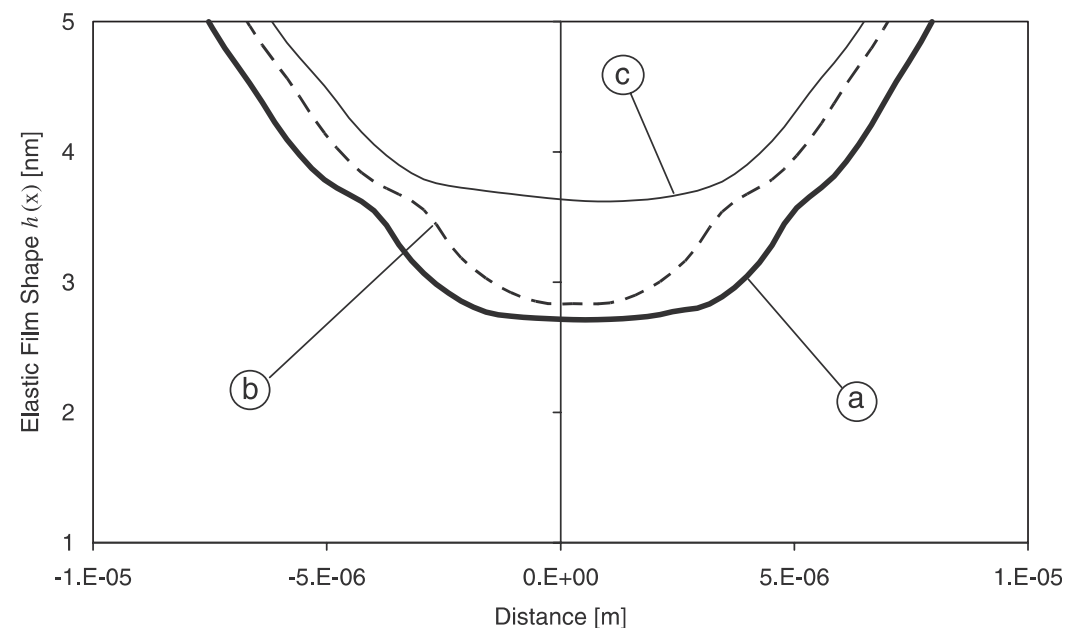

Fig. 3 The oil film thickness for three $\mathrm{h}_{\text {ref }}$

conjunction. When the load, and thus the stored energy, reaches certain discrete levels, then layering of film is noted in a step-wise fashion (see Fig. 2(b)). This finding is in line with those reported by Matsuoka and Kato [7] and Al-Samieh and Rahnejat [11].

At first sight, Fig. 2(b) suggests film thickness bifurcation at a given contact load or energy level. This is, in fact, not true, because the discrete number of molecular layers remains unaltered for a range of contact loads. Changes in contact load levels is, in fact, as the result of variations in strain energy tensor in the constraining solid surfaces, caused by their free surface energies (the solvation effect). A comparison of elastic gap shape corresponding to the minimum films at layer levels c, b, and a in Fig. 2 gives a clear picture of the underlying mechanism (see Fig. 3). Note the strain energy in the elastic film shape c in Fig. 3 is released to yield a lower contact load (see Fig. 2(b)) and a restoring contact geometry (Fig. 3, trace b) with a lower molecular level film. This action corresponds to the drainage of a layer of fluid from the contact. Thus, the released strain energy converts to the necessary kinetic energy to eject a molecular layer of the fluid. A further increase in load from position $b$ to $a$ increases the free surface energy interactions and results in new deformation of the contact (Fig. 3, trace a). Although further increases in the contact load, or an imposed reduction in separation, can, theoretically, cause further discretization in the fluid film, in practice, molecular films below two molecular levels are unlikely as this is the limit of adhesion, as noted by Bhushan [29] and Al-Samieh and Rahnejat $[\mathbf{1 1}, \mathbf{1 8}]$. This is also in line with the tenets of Newtonian viscous flow model (for wetted surfaces, at least one layer of molecule should adhere to each wall - this is physically ensured by the meniscus effect $[\mathbf{1 1}])$. The results show that, although the observed drainage of fluid from such contacts in discrete steps was noted by Chan and Horn [14] to be not in accord with the Newtonian model, this behaviour is in fact not in conflict with it. It merely indicates that the solvation effect becomes the dominant mechanism, as shown in Fig. 4. Note that the total pressure is dominated by the contribution due to solvation throughout the transition $c \rightarrow b \rightarrow a$, but the hydrodynamic effect remains undiminished throughout. The contribution due to van der Waals pressure represents merely one per cent of the total.

The fact that solvation pressure promotes surface deformation and recovery, associated with the kinetic energy of molecular re-ordering, means that the discretization process takes place with both principles of conservation of energy and momentum remaining intact (indicated by the arrow in Fig. 2(b)). Therefore, the belief that oscillatory density distribution near a constraining wall is structureless in nature is quite unfounded, and accounts for the one of the significant finds of the current work. This had arisen from the previous analyses by Matsuoka and Kato [7] and Abd-AlSamieh and Rahnejat [8] who had not taken into account the convergence of solvation pressure within the conjunction as a prerequisite in the mechanism of film formation.

\subsection{Transient}

In the preceding section, a series of pre-specified separations were investigated. The results imply that any gap size in excess of two molecular diameters of the intervening fluid can be attained by a combination of strain energy of surface deformation and molecular interactions, such that the total energy in the system follows the Euler relationship, $W=-\partial E / \partial z$. This, of course, ignores the stepped 

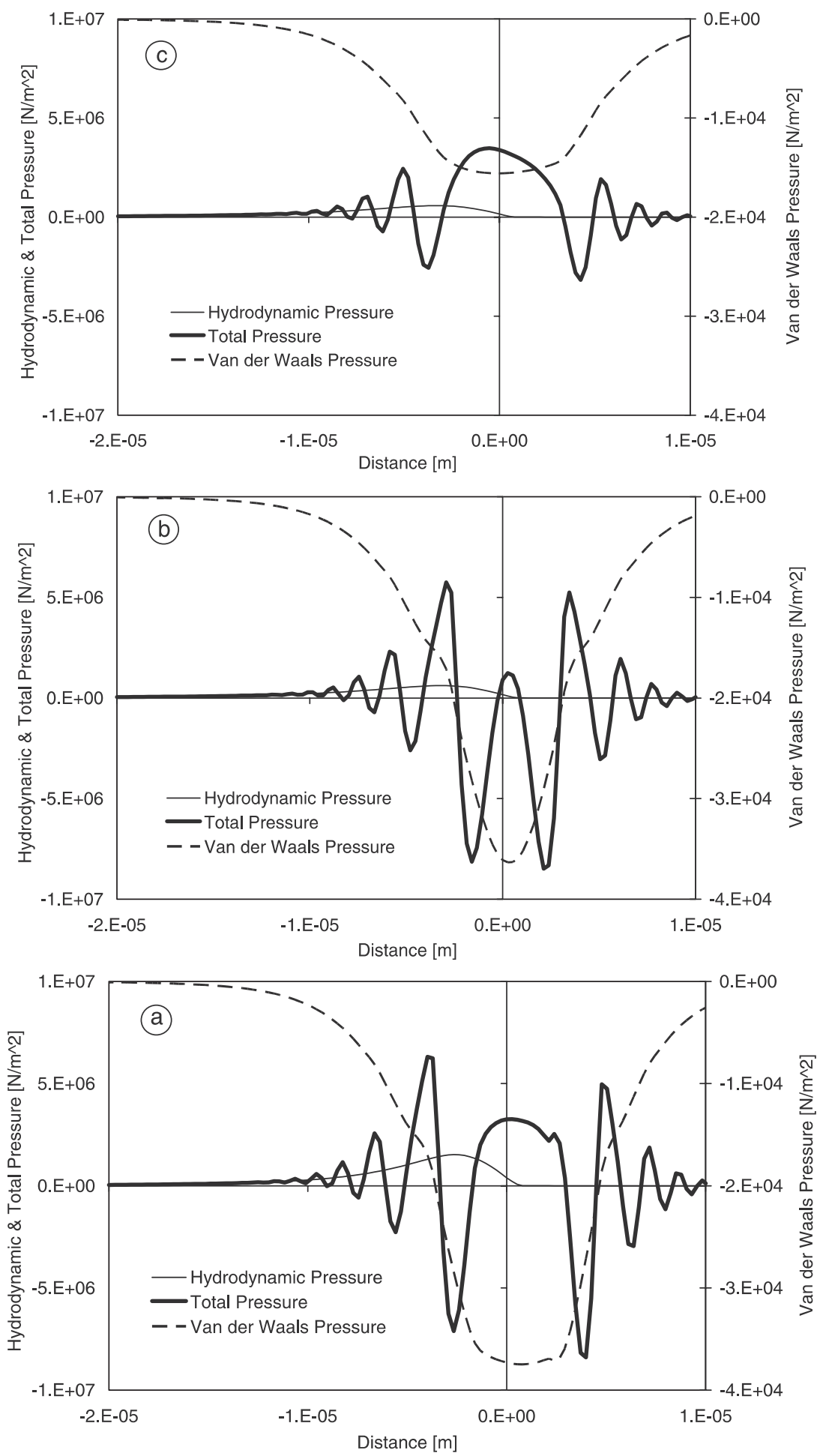

Fig. 4 Contributions to, and the total conjunctional pressure $\left(U_{a v}=2 e-4(\mathrm{~m} / \mathrm{s})\right)$

approach of the surfaces, which amounts to an applied momentum due to rigid body squeeze. For an equivalent roller near a semi-infinite elastic half-space, this rigid approach is the velocity of the centre of the roller, given by $\partial h_{r e f} / \partial t$. This amounts to an applied momentum (an inertial force, and additional energy input) to the conjunction which is unaccounted for in the initial study highlighted in the preceding section. Hence, pre-specifying a number of separations disregards the history due to inertial loading and a transient analysis is required to include it. For energy levels within the classical Hertzian impact, the contacting surface undergoes an elastic squeeze-film effect, which is at any location given by $\partial h_{x, t} / \partial t=\partial h_{r e f} / \partial t+\partial \delta_{x, t} / \partial t$, where the localized deformation is clearly in an opposite 
sense to the rigid approach (creating a deformation with sufficient load). Note that $\partial h / \partial t>0$, when $|\partial \delta / \partial t|>\left|\partial h_{\text {ref }} / \partial t\right|$ since the rigid squeeze $\partial h_{\text {ref }} / \partial t<0$ for approaching surfaces. Therefore, at each molecular level separation, there exists an elastic squeeze-film tensor, which, together with the hydrodynamic entraining motion, represents the local kinematic conditions. Therefore, to represent transient analysis one can impose a rigid body squeeze action, which in turn can also introduce local elastic deformation at any location $x, y$. Now, if one obtains a solution as highlighted by the flowchart of Fig. 1, the effect of inertial dynamics on contact behaviour becomes clear. Figure 5(a) shows the transition from the initial undeformed hydrodynamic behaviour (indicated in the figure by (d)) to that dominated by solvation through layering to the level indicated by $\partial h_{r e f} / \partial t=0$ for a quasistatic analysis (identical to the separation shown as (b) in Fig. 2(a)). Two theoretical inertial approaches at the same speed of entraining motion as the quasi-static case have been considered in Fig. 5(a). Note that increasing inertial approach inhibits the discretization effect and enhances separation. This is due to increasing influence of hydrodynamic contribution as shown in Fig. 6(a). Note that in this figure the pressure distribution for $\partial h_{r e f} / \partial t=0$ is dominated by solvation, whose contribution becomes less pronounced with the increasing value of $\partial h_{\text {ref }} / \partial t$. The pressure distributions in Fig. 6(a) correspond to the various separations denoted by (e) in Fig. 5(a). Figure 5(b) shows the increased load-carrying capacity of the contact with increasing squeeze effect, which is now well understood for conventional hydrodynamic contacts. Figure 6(b) shows the increased film thickness due to the inertial effect (squeeze-film action). Note that the deflection is caused in all cases by solvation, effectively introducing a separation rate $\partial \delta / \partial t$, discussed above.

The history of elastic film shape in Fig. 6(b) can be regarded as a wavefront, whose squeeze velocity varies from location to location ( $n$ points along the contact) at any instant of time. When a layer of molecules is drained through the contact, the wavefront accelerates at each locality according to $\beta=d / d t\left(\partial h_{x, t} / \partial t\right)$. Therefore, at any instant of time, according to the rigid approach of the roller, denoted by $\partial h_{r e f} / \partial t, \beta$ may be regarded as an equivalent gravitational effect, comprising the inertial effect, as well as the effect of contact pressure due to all the included mechanisms at work (in this analysis: hydrodynamics, solvation, and van der Waals interactions) [11].

Figure 7 shows the variation of $\beta$ at location (e) when $\partial h_{\text {ref }} / \partial t=0 \rightarrow 10 \mathrm{~nm} / \mathrm{s}$. The sharp drop in value of $\beta$ around the central region of the contact is due to elastic deformation there (see Fig. 6(b)),
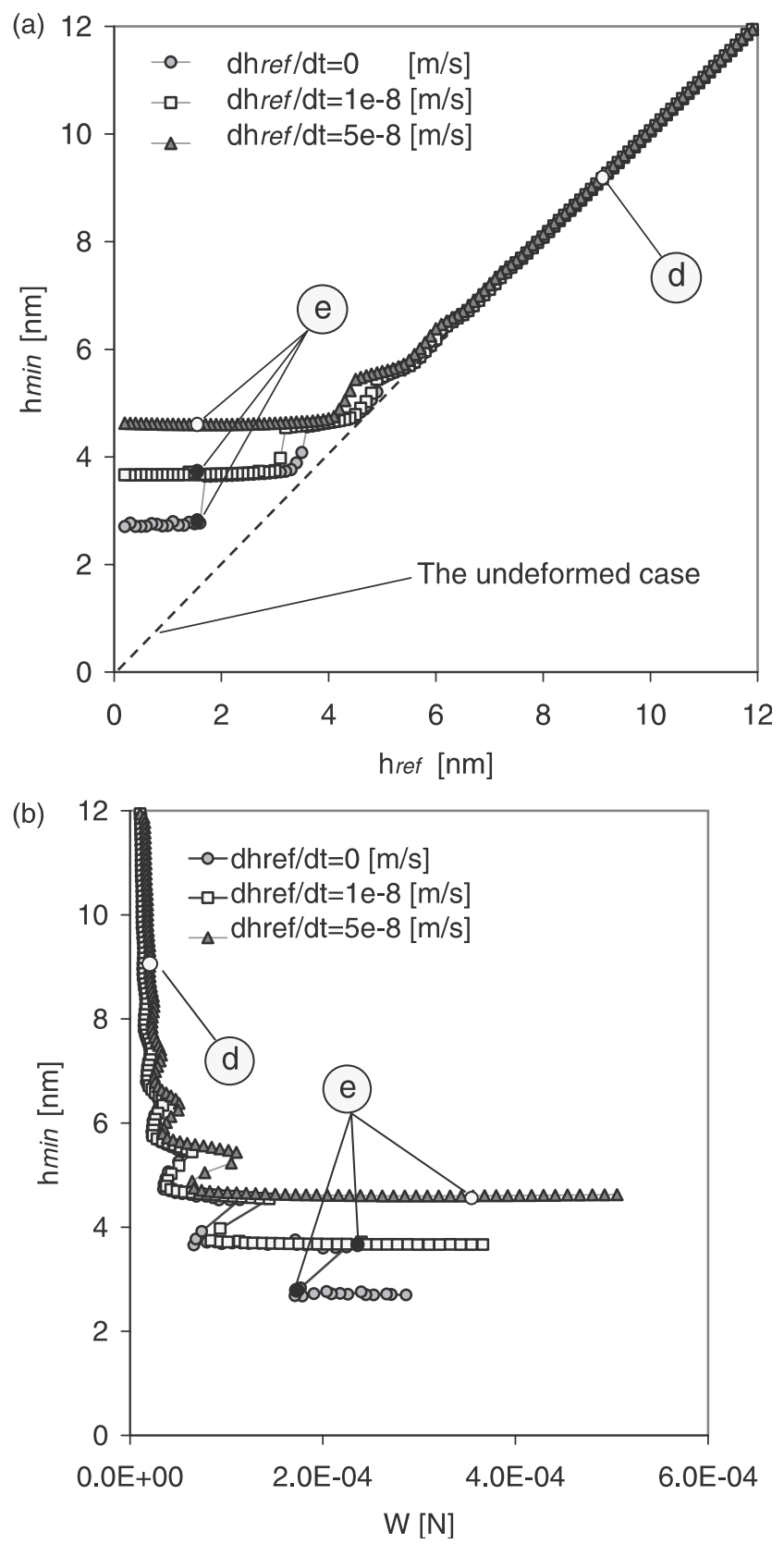

Fig. 5 Minimum film thickness for various squeeze-film velocities

and may be viewed as an equivalent induced local gravitational effect. The implication is that kinetic energy introduced by the imposed rigid body squeeze motion of the roller (i.e. rate of convergence of the contiguous surfaces) can only take place in a constrained configuration space determined by the combined forms of acting kinetics in the contact (i. e. in $r$ coordinates) with $n$ particles representing the system of surfaces and the intervening fluid molecules as a many-body system.

Therefore, the kinetic energy for such $n$ particles of mass $m_{k}$ yield accelerations such as $\beta$, described 

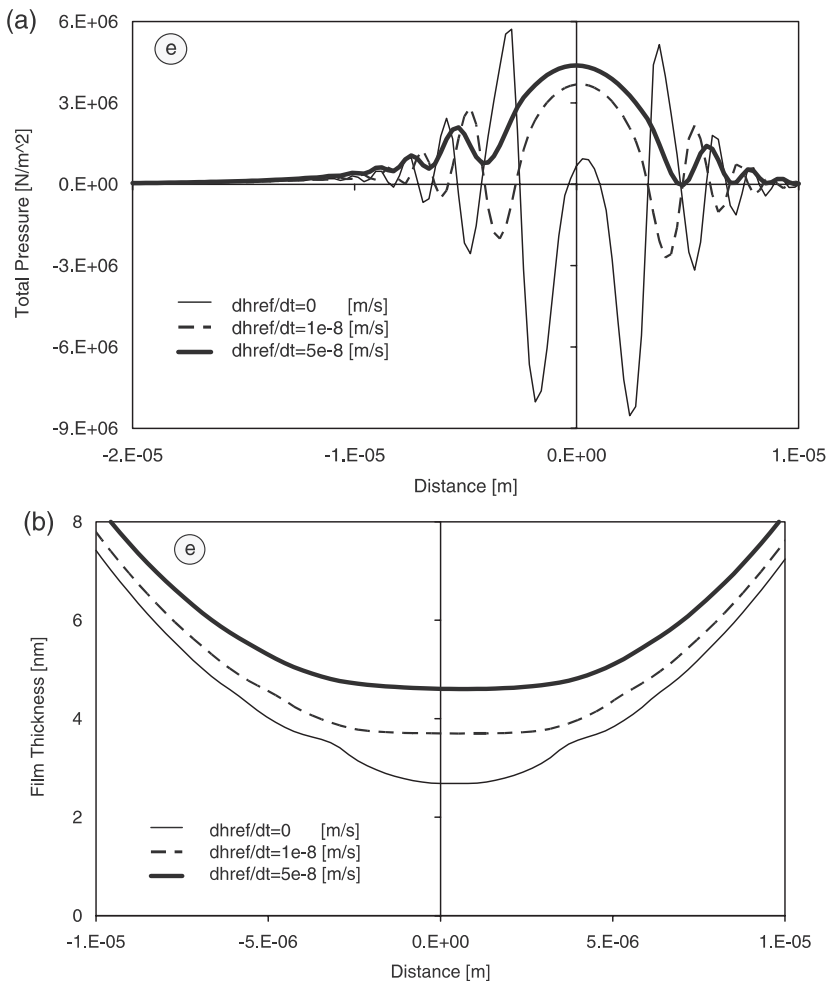

Fig. 6 Pressure distribution and film shape with different squeeze velocities: (a) pressure distribution, (b) elastic film shape

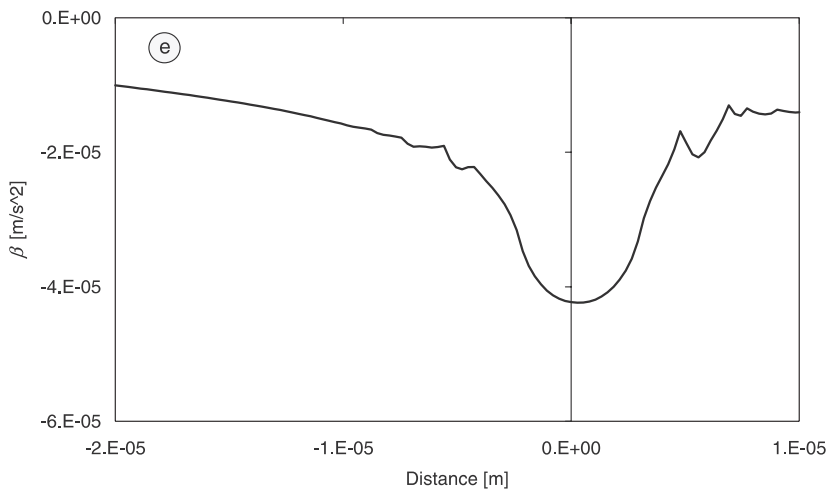

Fig. 7 Equivalent gravitational action for the position (e) in Fig. 5(a), when $\frac{d h_{r e f}}{d t}=1 \times 10^{-8} \mathrm{~m} / \mathrm{s}$

above, to oppose the applied inertial force (i.e. the D'Alembert principle). Thus, using the usual tensor notations $\partial h_{r e f} / \partial t=x_{i} \cdot \dot{q}^{i}$, and hence $K_{k}=$ $1 / 2 m_{k} x_{i} \cdot x_{j} \dot{q}^{i} \dot{q}^{j}=1 / 2 m_{k} \mathbf{g}_{i j} \dot{q}^{i} \dot{q}^{j}$, where $g_{i j}=x_{i} \cdot x_{j}$ is the metric tensor, being intrinsic to the local condition of particle of mass $m_{k}$. Let $M_{i j}=g_{i j} m_{k}$ and, therefore, for the whole many-body system $K=$ $1 / 2 \sum_{i j} M_{i j} \dot{q}^{i} \dot{q}^{j}$. The applied conjugate momentum is then obtained as $p_{i}=\partial K / \partial \dot{q}^{i}=\sum_{i} \mathrm{M}_{i j} \dot{q}^{i}=\dot{q}_{i}$, which must be covariant and represent the change in configuration space during the applied motion (i.e. due to combined action of all the forces: hydrodynamic, van der Waals, and solvation). Lagrange's equation for the generalized coordinate, $q_{i}$ in terms of absolute derivative becomes

$$
\frac{D \dot{q}_{i}}{D t}+\frac{\partial U}{\partial q^{i}}=0
$$

where $\frac{D \dot{q}^{i}}{D t}=\frac{d \dot{q}_{i}}{d t}-\frac{1}{2} \frac{\partial \mathrm{M}_{j k}}{\partial q^{i}} \dot{q}^{j} \dot{q}^{k}$.

Note that $-\partial U / \partial q_{i}=F_{i}$ (Euler's equation), which is the net contact force. Thus, $D \dot{q}_{i} / D t=F_{i}$.

This shows that the absolute derivative of the velocity equates to the net force, and thus the action of all the involved kinetics can be replaced by an equivalent gravitational field. This field is determined by the potential energy deforming the surfaces and determining the gap between them, the constraining effect of which governs the discrete behaviour of surface films of molecular dimension. If the value of the absolute derivative falls to zero, then the motion is one of free fall (i.e. rigid body action in this case, or 'geodesic'). This is a major find, because it shows that the little understood nano-tribological contacts can actually be classified by the absolute derivative of velocity within continuum mechanics.

For two dimensional analysis considered here, the maximum shear stress field below the surface is obtained as ${ }_{k} \sigma_{1}=\left[\left({ }_{k} \sigma_{x x}-{ }_{k} \sigma_{y y}\right)^{2}+4_{k} \sigma_{x y}^{2}\right]^{1 / 2}$. Within elastic limit, its absolute maximum value, in accord with the Hertzian theory, is $0.3 P_{H}$, where $P_{H}$ is the maximum Hertzian pressure. The orthogonal shear stress $\left({ }_{k} \sigma_{x y}\right)$ is predicted to be zero along the centreline axis of the sub-surface stress field beneath the contact surface, and has opposite signs on its either sides. Lundberg and Palmgren [30] considered the maximum reversing orthogonal shear stress to be responsible for the fatigue life of contacting surfaces, since the double amplitude of this stress is in fact larger than the magnitude of the absolute maximum shear stress.

Figure 8 shows the maximum and orthogonal shear stress fields for the contact conditions corresponding to the various separations denoted by (e) in Fig. 5(a). The corresponding pressure distributions are shown in Fig. 6(a). Figure 8(i) corresponds to the highest value of the inertial approach $(\partial h / \partial t=5 E-8)$. This condition corresponds to dominant rigid body inertial effect, where the squeeze-film action plays the major role. It gives rise to increasing hydrodynamic pressure, and due to increased load-carrying capacity, maintains a relatively thick film. The solvation contribution is relatively low. Thus, the induced sub-surface stress fields are relatively symmetrical with small ripples generated by slowly rising solvation pressure. Reduced squeeze action diminishes the inertial contribution and the surfaces converge, leading to dominance of solvation and molecular layering of the film. The oscillatory behaviour of the pressure 
(i)

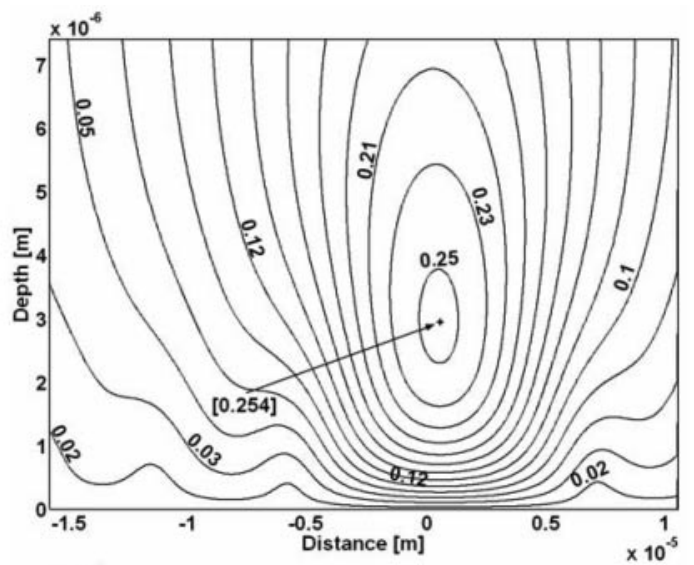

(ii)

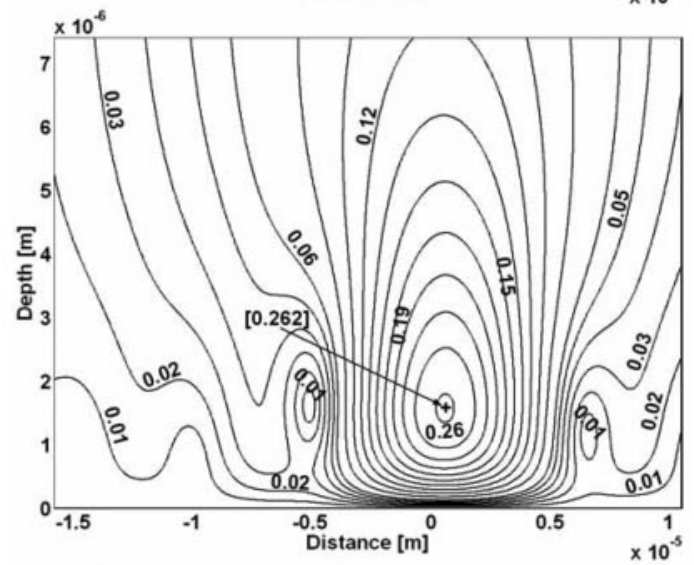

(iii)

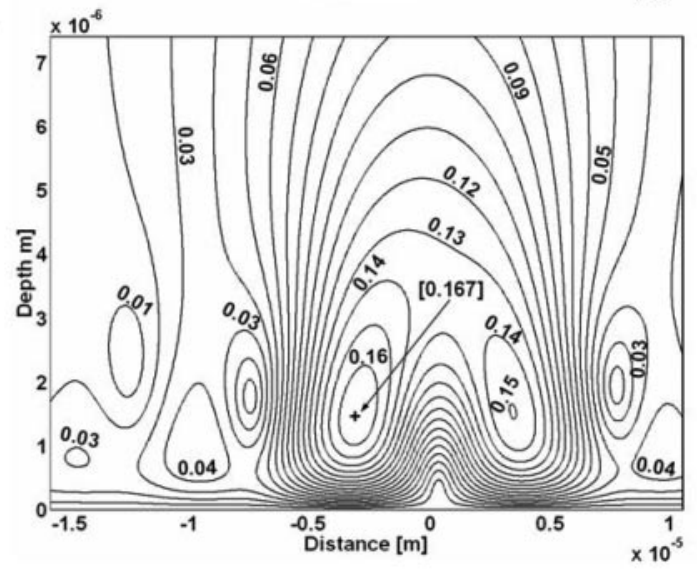

Maximum shear stress tensor field
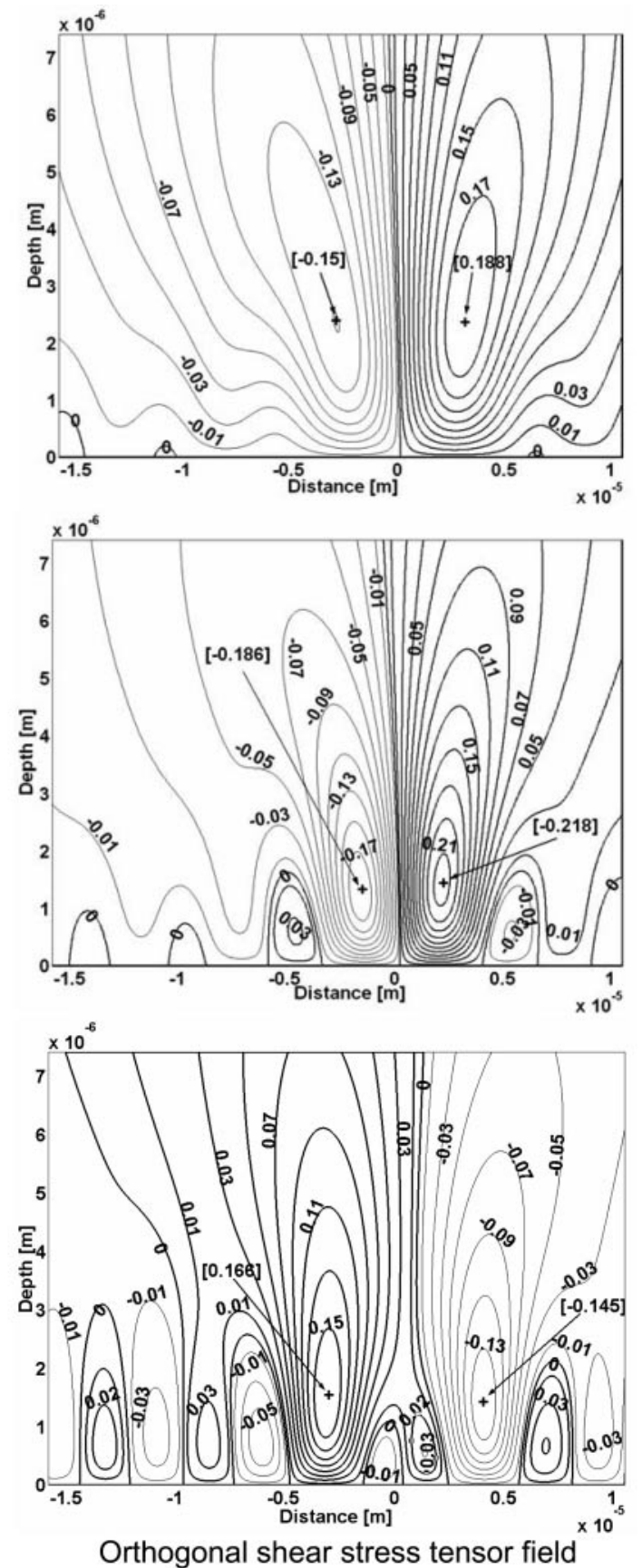

Fig. 8 Subsurface stress field for the pressure cases shown in Fig. 6(a) (i) $\partial h / \partial t=5 E-8$; (ii) $\partial h / \partial t=E-8$; (iii) $\partial h / \partial t=0$

distribution translates into oscillations in the subsurface stress field. Both the absolute maximum shear stress and reversing orthogonal shear stress peak values migrate towards the contact surface, with the implication of greater chance of surface distress and cracking. The growing number of stress field isoclines in Figs 8(ii) and 8(iii) show this trend. The reversing nature of very localized shear stresses is indicative of waveform distortion at contact surface and in the layers beneath it caused by the dominant oscillatory pressure behaviour. A practical implication of this for vanishing gaps of a few to several molecular diameters of an intervening fluid formed between thinly coated surfaces or wafer thin structures is the increased chance of delamination. Note that many magnetic storage devices use both protective, as well as magnetic layers, and there would be implications for their further miniaturization, especially for space and military applications.

\section{CONCLUSION}

The study proposes an integrated methodology for predicting the fluid film pressure, the local pressure 
generated by the molecular interactions, local elastic deflection and subsurface stress field for nano-scale contacts. It is proven that, for fast inertial approaches, the ultra-thin film thickness is governed by the squeeze-film effect, and for slow inertial approaches, by the molecular level interactions. The immediate implication of these findings for practical applications (e.g. hard disk drives or MEMS devices) is that an accurate prediction should consider the dynamics of the micro-scale mechanism as well as the reaction generated by the ultrathin film within a common framework. These combined phenomena govern the squeeze-film effect, and, therefore, prohibit the ultra-thin film from decreasing below several layers of molecules. Predicting the subsurface stress field is particularly important for very thin films where the pressure oscillations generated by the solvation effect govern the local pressure distribution. In response, the highest peak of the maximum shear stress field migrates closer to the surface and the number of reversals in the orthogonal shear stress field increases significantly. Most micro-scale devices have at least one of the approaching surfaces made of silica, which is a brittle material, and these phenomena have major implications towards localized fatigue and surface degradation. The paper introduces an alternative method of investigating the local elastic deflection of the approaching bodies. Starting from the D'Alembert principle, which states that the kinetic energy of all the particles involved equals the applied force, an equivalent gravitational field was found. This field is generated by the potential energy responsible for the localized deflection of the surfaces, and, therefore, governs the size and the discrete behaviour of the resulting film thickness.

\section{REFERENCES}

1 Reynolds, O. On the theory of lubrication and its application to Mr Beachaump Tower's experiments including an experimental determination of the viscosity of olive oil. Phil. Trans. R. Soc., Ser. A, 1886, 177, 157.

2 Lifshitz, E. M. The theory of molecular attractive forces between solids. Soviet Phys. JETP (Engl. Transl.), 1956, 2, 73-83.

3 Dzyaloshinskii, I. E., Lifshitz, E. M., and Pitaevskii, L. P. The general theory of van der Waals forces. $A d v$. Phys., 1961, 10, 165-209.

4 Derjaguin, B. V. and Landau, L. Theory of the stability of strongly charged lyophobic sols and the adhesion of strongly charged particles in solutions of electrolytes. Acta. Physicochim. USSR, 1941, 14, 633-662.

5 Verwey, E. J. W. and Overbeek, J. Th. G. Theory of Stability of Lyphobic Colloids, 1948 (Elsevier, Amsterdam).

6 Israelachvili, J. N. Intermolecular and Surface Forces, 1992 (Academic Press, New York).
7 Matsuoka, H. and Kato, T. An ultra-thin liquid film lubrication theory - calculation method of solvation pressure and its applications to EHL problem. Trans. ASME, J. Tribology, 1997, 119, 217-26.

8 Abd-AlSamieh, M. F. and Rahnejat, H. Nano-lubricant film formation due to combined elastohydrodynamic and surface force action under isothermal conditions. Proc. IMechE Part C: J. Mech. Eng. Sci., 2001, 215, 1019-29.

9 Tichy, J. A. A surface layer model for thin film lubrication. ASLE Trans., 1995, 38(3), 577-582.

10 Tambe, N. S. Nanotribological investigations of materials, coatings and lubricants for nanotechnology applications at high sliding velocity. $\mathrm{PhD}$ Thesis, Ohio State University, 2005.

11 Al-Samieh, M. F. and Rahnejat, H. Physics of lubricated impact of a sphere on a plate in a narrow continuum to gaps of molecular dimensions. J. Phys. D: Appl. Phys., 2002, 35, 2311-2326.

12 Ashurst, W. R., Carraro, C., and Maboudian, R. Vapor phase anti-stiction coatings for MEMS. IEEE Trans. Device Mater. Reliab., 2003, 3(4), 173-178.

13 Kobrin, B., Chinn, J. and Ashurst, W. Durable antistiction coatings by molecular vapor deposition (MVD). NSTI-Nanotech, 2005, 2, 347-350.

14 Chan, D. Y. C. and Horn, R. G. The drainage of the thin liquid films between solid surfaces. J. Chem. Phys., 1984, 83, 5311-24.

15 Bhushan, B. Handbook of Micro/Nanotechnology, 2nd edition, 1999, (CRC Press, Boca Raton, Florida).

16 Maboudian, R. and Carraro, C. Surface chemistry and tribology of MEMS. Annu. Rev. Phys. Chem., 2004, 55, 35-54.

17 Tanner, D. M., et al. MEMS reliability: infrastructure, test structures, experiments, and failure modes. Sandia Report, SAND2000-0091, pp. 31, 2000 (Sandia National Laboratories).

18 Al-Samieh, M. F. and Rahnejat, H. Ultra-thin lubricating films under transient conditions. J. Phys. D: Appl. Phys., 2001, 34, 2610-21.

19 McGuiggan, P. and Israelachvili, J. N. Adhesion and short-range forces between surfaces. Part II: effects of surface lattice mismatch. J. Mater. Res., 1990, 5(10), 2232-2243.

20 Roelands, C. J. A. Correlation aspects of viscositytemperature-pressure relationship of lubricating oils. PhD Thesis, Delft University of Technology, The Netherlands, 1966.

21 Dowson, D. and Higginson, G. R. Elasto-Hydrodynamic Lubrication, 1966, (Pergamon Press Ltd, Oxford).

22 Horn, G. and Israelachvili, J. N. Direct measurement of structural forces between two surfaces in a nonpolar liquid. J. Chem. Phys., 1981, 75, 1095-1102.

23 Hamrock, B. J. Fundamentals of Fluid Film Lubrication, 1994 (McGraw-Hill Companies, New York, London, New Mexico).

24 Christenson, H. K. Capillary condensation in systems of immiscible liquids. J. Colloid. Interface Sci., 1985, 104(1), 234-249.

25 Teodorescu, M., Rahnejat, H., and Gohar, R., Harmonic analysis to determine contact characteristics of concentrated counterformal contacts. In Proceedings 
of 7th Biennial ASME Conference on Engineering Systems Design and Analysis, Manchester, UK, July 19-22, 2004.

26 Teodorescu, M., Rahnejat, H., Gohar, R. and Dowson, D. Harmonic decomposition analysis of contact mechanics of bonded layered elastic solids. In review process, Proc. IMechE, J. Mech. Eng. Sci., 2006

27 Hertz, H. On the contact of elastic solids., J. Reine Angew. Math., 1881, 92, 156-157.

28 Barber, J. R. Elasticity, 2nd Edition, 2002 (Kluwer Academic, Boston and London).

29 Bhushan, B. Contact mechanics of rough surfaces in tribology: multiple asperity contact. In Tribology of Magnetic Storage Systems (Ed. B. Bhushan), 1998, pp. 123-60 (John Wiley \& Sons, New York).

30 Lundberg, G. and Palmgren, A. Dynamic capacity of rolling bearings. Acta. Polytechnica, Mech. Eng. Series I, 1947, Royal Swedish Academy of Engineering Sciences, 1, 3, 1-50.

\section{APPENDIX}

\section{Notation}

a molecular diameter of fluid

$A_{131}$ Hamaker constant

$C$ constant used in defining the solvation pressure

$D \quad$ influence coefficient matrix

E total energy

$F_{m} \quad$ meniscus force

$g_{i j} \quad$ metric tensor (first fundamental form)

$h$ film thickness

$h_{\text {min }}$ minimum film thickness

$h_{\text {ref }}=h_{0 \mid x=0}$ minimum undeformed separation

$k$ Boltzmann constant, and harmonic order when a subscript

$K \quad$ kinetic energy $m_{k} \quad$ particle mass in many-body interactions

$M_{i j} \quad m_{k} g_{i j}$

$p_{i} \quad$ conjugate momentum tensor

$P \quad$ total conjunctional pressure

$P_{0} \quad$ constant average pressure

$P_{h} \quad$ hydrodynamic pressure

$P_{H} \quad$ maximum Hertzian pressure

$P_{k} \quad$ amplitude of the $k^{t h}$ harmonic of the applied pressure $k=1 \rightarrow N$

$P_{s} \quad$ solvation pressure

$P_{w} \quad$ van der Waals pressure

$q^{i}, q^{j} \quad$ generalized coordinates

$s \quad$ profile of contacting solids

$t$ time

$T$ temperature

$u_{a v} \quad$ speed of entraining motion

$U$ potential energy in configuration space

$W$ applied load

$Z \quad$ viscosity-pressure index

$\alpha \quad$ lubricant piezo-viscosity index

$\beta \quad$ local gravitational acceleration

$\gamma_{L} \quad$ surface energy of liquid

$\delta \quad$ local deflection

$\varepsilon, \xi \quad$ constants used in defining the bulk density of fluid

$\zeta \quad$ equivalent damping factor

$\eta \quad$ dynamic viscosity of lubricant

$\eta_{0} \quad$ dynamic viscosity at atmospheric pressure

$\theta \quad$ contact angle

$\lambda_{k} \quad$ wavelength for the $k^{\text {th }}$ harmonic

$\rho_{\infty} \quad$ bulk density of lubricant

$\rho_{0 \infty} \quad$ atmospheric density of fluid

${ }_{k} \sigma_{i j} \quad k^{t h}$ harmonic of stress tensor $\quad k=0 \rightarrow N$

$\varphi_{k} \quad$ phase angle for the $k^{t h}$ harmonic

$\omega_{d}$ equivalent damped natural frequency

$\omega_{n} \quad$ equivalent undamped natural frequency 